\title{
Fluorine F18 T807
}

National Cancer Institute

\section{Source}

National Cancer Institute. Fluorine F18 T807. NCI Thesaurus. Code C131212.

A radioconjug ate composed of the paired helical filament (PHF) tau binding agent T807, a benzimidazole pyrimidine derivative, conjug ated to the radioisotope fluorine $F 18$, with potential tau positron emission tomography (PET) imaging activity. Upon administration of fluorine F18 T 807, the T807 moiety targets and binds to PHF-tau in the brain, which allows PET imaging of PHF-tau and analysis of phosphorylated PHF-tau aggregates (neurofibrillary tang les). Increased levels of PHF-tau and neurofibrillary tangles are correlated with the cognitive decline seen in neurodegenerative diseases, such as dementia, Alzheimer's disease (AD) and chemotherapy-induced cognitive impairment $(\mathrm{ClCl})$. 